\title{
Reliable and Power Efficient Routing Protocol for MANETs
}

\author{
V.Sowmya Devi \\ Assistant Professor, Dept. of CSE, GITAM University, Hyderabad, India. \\ E-mail:vallala.sowmya@gmail.com \\ Dr. Nagaratna P Hegde \\ Professor, Dept. of CSE, Vasavi College of Engineering Hyderabad, India.
}

\begin{abstract}
In this paper, we focused on of the most proliferated network that is Mobile Adhoc Network (MANET). Due to the dynamic nature and limited power of nodes, the routes will fail frequently which intern cause high power dissipation. This paper proposed a reliable and power efficient routing with the nodes having high power level. As well as, this approach also concentrated on the reduction of power consumption during route failures by adapting an on-demand local route recovery mechanism through a set of helping nodes and they are called as Support Nodes (SN). The cooperation of support nodes will reduce the power consumption and significantly increases the reliability. The performance of proposed approach was evaluated through average energy consumption, packet delivery ratio and end-to-end delay over varying node speed and varying packet size. The power optimization and reliability achieved by the proposed approach gives an ideal solution to the future communication in MANETs for a long time.
\end{abstract}

Index Terms-MANETs, Power efficiency, reliability, support nodes, Packet Delivery Ratio, Delay.

\section{INTRODUCTION}

MANET (Mobile Adhoc Network) [1] is one of the Adhoc network type that uses mobile devices as nodes for the purpose of communication through the wireless links which are relatively bandwidth constrained. Since the nodes in MANET are mobile devices, the topology changes of the network are very rapid in nature and it is not-predictable over time. Due to the dynamic network topology, shortage power of mobile nodes and timevarying wireless channels, routing protocols design and communication process for this type of networks is becoming an exciting problem. Due to these characteristics, the routes of MANET are unstable and causing routes to break frequently which consumes more energy and time. Hence, there is a need of an efficient routing protocol which provides timely and reliable data delivery through minimum resource utilization. The routing protocols proposed for MANETs are generally categorized as table-driven and on-demand driven, based on the timing of when the routes are updated. Many routing protocols including Destination-Sequenced Distance Vector (DSDV) [2] and Fisheye State Routing (FSR) protocol [3] belong to this table driven category, and they differ in the number of routing tables manipulated and the methods used to exchange and maintain routing tables. In contrast totable-driven routing protocols, not all up-to-date routes are maintained at every node. Dynamic Source Routing (DSR) [4] and AdHoc On-Demand Distance Vector (AODV) [5] are examples of on-demand driven protocols. In contrast to simply establishing correct and efficient routes between pair of nodes, one important goal of a routing protocol is to keep the network functioning as long as possible. As discussed earlier, the main aim of this approach is to design a new routing protocol by minimizing resource consumption and also to increase reliability of data delivered.

In this paper, a new routing protocol named Power Efficient Reliable Routing (PERR) was proposed for MANETs. This routing protocol tries to construct a route based on the capability of neighbor node. The capability of neighbor nodes was measured by Power-Reliability (PR) metric. The PR metric is the combination of nodes remaining energy and reliability. Here, the successful packet transfer capability was considered as reliability. By combining node's residual energy and reliability, this approach achieves both high energy efficiency and reliable packet delivery. This approach also allows to local route recovery during route failure through supporting nodes. Thus, the proposed approach constructs a reliable route that will ensure balanced energy consumption by reducing the control messages of the mobile node in MANET environment. The rest of the paper is organized as follows: section II illustrates the details about the earlier proposed approaches. Section III gives the details about the evaluation of PR metric. Section IV illustrates the details about the proposed approach. Section V illustrates the simulation results and finally section VI concludes the paper.

\section{RELATED WORK}

Various approaches were proposed in earlier to perform energy optimization of MANETs. AODV [5] is a basic reactive routing protocol which constructs a route 
on demand basis, i.e., the routes are constructed whenever they needed and maintained as long as they required. The route construction process of AODV consumes more energy since; it uses flooding transmission of RREQ, RREP, or HELLO messages. Due to the complicated route failures, this approach consumes more time and effort. Without changing the route discovery process, [6] proposed an enhancement to AODV and named as AODV with backup routing (AODV-BR). In AODV-BR, every node creates its own alternate routes by overhearing the RREP messages of destination. In the case of detection of a route failure, the node which detects the route failure broadcasts the data packets to its one-hop neighbors. Once the data received at one-hop neighbors, they forward the data towards the destination through the pre-established back up route. AODV-BR focused on reliability only, thus it establishes delayed routes. The main drawback of AODV-BR is high end-to-end delay due to delayed routes and also dissipation of high energy. In [7], an adaptive backup routing based AODV was proposed which is similar to AODV-BR. This approach reconstructs a route by considering multiple backup routes from immediate upstream node through the process of three-way handshaking during route failure. This approach can't escape from collisions due to the consideration of multiple backup routes. By considering a back-off timer, [8] proposed an approach which was just improvement to AODV-ABR, named as IBR-AODV. IBR-AODV performs the local route recovery by considering a back off timer and handshaking process. This approach tries to minimize flooding of control messages and also collisions. The main drawback of this approach is huge energy consumption for the pro-active back routing concept. Robust and reliable routing [9] used link stability metric to construct a new route. Node's mobility, residual energy and data delivery are used in the evaluation of link stability metric. Link stability based routing [9] selects an intermediate node which is having minimum level of link stability metric for RREQ forwarding. Thus, R2-routing constructs a route with minimum stability nodes. In [10], the intermediate node selects a route having highest and robust route index (RRI) value among the multiple route requests received. RRI is evaluated from the navigated hop length, corresponding node speed and node delay. This approach is more effective for highly mobile scenarios. The main drawback of this approach is high protocol complexity because; the node needs to retrieve its current parametric value and its current position from different layers. Path encounter rate (PER) [11] is a routing metric measured as the sum of square average encounter rate. PER based routing protocol tries to select a route which is having minimum encounter rate value among the available paths towards the destination node. PER well suited for the nodes with less mobility or for low node density regions. This routing approach tries to pass entire traffic load through a single and specific region, which creates vulnerabilities and also increases the collision probability. There is some other path duration metrics [12-14] proposed with the view of encounter based metric. In energy aware routing [15], a node forwards the route request message only if it has energy more than a predefined threshold value. Else, it drops the RREQ message. This type of routing approach overcomes the problem of overhead during periodic exchanges, but, in the case of route failure, it suffers from more end-to-end delay to reconstruct a route. AODV-PE [17], EAODV [16] and energy efficient reliable routing [18] are some other energy aware routing protocols which gives higher lifetime for a network by selecting a route having maximum energy. But, a node with limited energy may exist in the maximum energy route, which increases the probability of route failure. Local energy aware routing protocols do not concentrate on the local route recovery since it reduces the packet delivery ratio and throughput of the network significantly. Energy efficient probabilistic routing (EEPR) protocols [19, 20, 21] significantly reduces the route request message propagation through their proposed metric. EEPR protocols do not consider the reliability of route and link quality. They do not consider link quality in terms of reliability of the route, which drastically reduces the network lifetime and data transfer efficiency by wasting the residual energy of the nodes having poor link quality. Even though EEPR [21] was proposed to significantly reduce the RREQ message propagation by choosing a metric that combines the node's ETX value with the node's residual energy value our simulation results show that the combined metric $[19,20,21]$ has almost no impact on the node's link quality.

\section{POWER-RELIABILITY METRIC}

Given a network with $\mathrm{N}$ number of nodes, a distinct identifier address $(i)$ was assigned to each and every node $i$ in the network. Let $P_{n \text { max }} n \in N$ and $P_{n}$ be the initial power and remaining power of node $n$. In the network any node requires $P_{\mathbf{p}, n}$ amount of power to transmit a data packet to its neighbor node $m$ and given by the following expression [22],

$$
P_{\mathbf{p}, n}=P_{r x(\mathbf{p}, n-1)}+P_{t x(\mathbf{p}, n)}
$$

Where, $P_{r x(\mathbf{p}, n-1)}$ is amount of power required to receive packet ' $\mathbf{p}$ ' from upstream node $n-1$ and $P_{t x(\mathbf{p}, n)}$ is the amount of power required to transmit packet ' $\mathbf{p}$ ' to downstream node $m$.

Reliability is one of the performance metrics which can give the information about the packet forwarding capability of a node in MANET. This can be measured as the ratio of actually received packet from neighbor node to actually sent packet to the neighbor node. This can also be termed as a packet delivery ratio (PDR) for a particular hop. Here, this evaluation considered the sum of data packets and control packets (Excluding HELLO packets) received from downstream node and sent to downstream node. Let node $n$ and node $m$ be the two communicating nodes, $S_{\text {Pnm }}$ be the packets sent from 
node $n$ to its one-hop downstream node $m$ and $R_{\mathbf{P} m n}$ be the Packets received at node $n$ from its one-hop downstream node $m$ at any time interval $t$. Then the reliability between node $n$ and node $m$ can be measured as

$$
R_{n(t)}=\frac{R_{\mathbf{p} m n}}{S_{\mathbf{p} n m}}
$$

In the time instant $t$, node $n$ simultaneously communicates with other neighbor nodes. Let $S_{\boldsymbol{P} i}$ be the packets sent from node $n$ to its other neighbor nodes $i$ and $R_{\boldsymbol{P} i}$ be the packets received at node $n$ from its other neighbor node $i$ at a time instant $t$. then the weighted reliability of node $n$ can be evaluated as,

$R_{n(t)}^{W}=(1-\alpha) R_{n(t)}+\alpha \max \frac{R_{P j}}{S_{P j}}, 1 \leq j \leq i, j \neq m$

Where $\alpha=$ weight factor $(0 \leq \alpha \leq 1)$.

From the above analysis, the power-reliability metric for node $n$ can be evaluated as,

$$
P R_{n}=P R_{n, \min }+\left(1-P R_{n, \min }\right) *\left(\frac{P_{n} * R_{n(t)}}{P_{n, \max } * R_{n(t)}^{W}}\right) * 1+\beta
$$

$$
\text { Where } \beta=\left(\sqrt[\gamma]{\frac{\left(R_{n(t)}-R_{n(t)}^{W}\right) * P_{\mathbf{p}, n}}{\left(1-R_{n(t)}^{W} P_{\mathbf{p}, n}\right)}}\right) \text { is a constant, varies }
$$

with the weighted reliability. In the above expressions, $P R_{n, \min }$ and $\gamma$ are predefined minimum $P R_{n}$ and weigh factor. Here, the power reliability metric will decide whether the node is able to forward data or not. A node can be selected for data transfer if it satisfies the following both conditions.

1. The node has sufficient power to receive and forward data from other neighbor nodes.

2. The node is capable (in the view of reliability) to transmit data to other nodes.

Combined power reliability routing metric will guarantee the nodes ability to transfer or send the data to other nodes without any failure, if it fulfills the following constraint,

$$
P R_{i}>P R_{i, m i n}, i \in N, i \neq \text { source and } i \neq \text { destination }
$$

Thus, for any node, the current PR value should be above the minimum PR value. Then only the node will be allowed for communication.

\section{PROPOSED APPROACH}

The complete proposed work is carried out in two phases such as route discovery and route maintenance.

\section{A. Route Discovery}

In this phase, like AODV, when a source node has data and wants to transmit to a destination node, it broadcasts a Route Request (RREQ) packet to its one hop neighbor node by setting the support node's address as null. Here support node is defined as the node which is able to reestablish a connection between the predefined source and destination pair in case of route failure. Initially, every node will establish a set of support nodes to overcome the problem of route failure. After the reception of RREQ at neighbor node, it checks for duplicity and performs the similar action to AODV. Then it searches for a routing path in its routing table towards destination. In this phase, the flag state will be mentioned in Table.1.

Table.1. Routing Flag

\begin{tabular}{|c|c|}
\hline State & Value \\
\hline Inactive & 0 \\
\hline Active & 1 \\
\hline Repair & 2 \\
\hline
\end{tabular}

If the neighbor node founds any routing information, then it will give a reply to source node through a Route Reply (RREP) packet and evaluates its PR metric, otherwise, it simply discards the RREQ. If the obtained PR metric was above the predefine threshold, the neighbor node forwards the RREQ. If the source node does not find any routing path information, then it will evaluate its own PR metric using (4). If the obtained PR metric value is greater than the predefined threshold (Threshold defined to control the dynamicity of network), then it will rebroadcast the RREQ towards the destination. After the reception of RREQ at destination node, it will give reply to source node through RREP packet. When an intermediate node receives the RREP packet, then it updates the support node's address from the RREP and also updates its routing table. The null space kept for support node was filled by the address of next hop node. This will continue at each and every intermediate node before the RREP reaches to source node. For example, in Fig.1, source node $S$ wants to send the information to destination $\mathrm{D}$, so it requires a route towards $\mathrm{D}$. then it broadcasts a RREQ packet into the network. Node A receives the RREQ and checks for destination and then rebroadcasts towards destination $\mathrm{D}$. Node $\mathrm{B}, \mathrm{P}$ and $\mathrm{M}$ receives the RREQ, only node $B$ rebroadcasts the RREQ because of low energy level of node $M$ and node $P$. In this fashion remaining node also broadcasts the RREQ until it reaches to destination. After receiving RREQ at destination it replies with a RREP. Intermediate nodes receive the RREP and then update their routing table with supporting nodes address. Source node receives the RREP and starts to send data to destination through the established path S-A-B-C-E-H-D. 


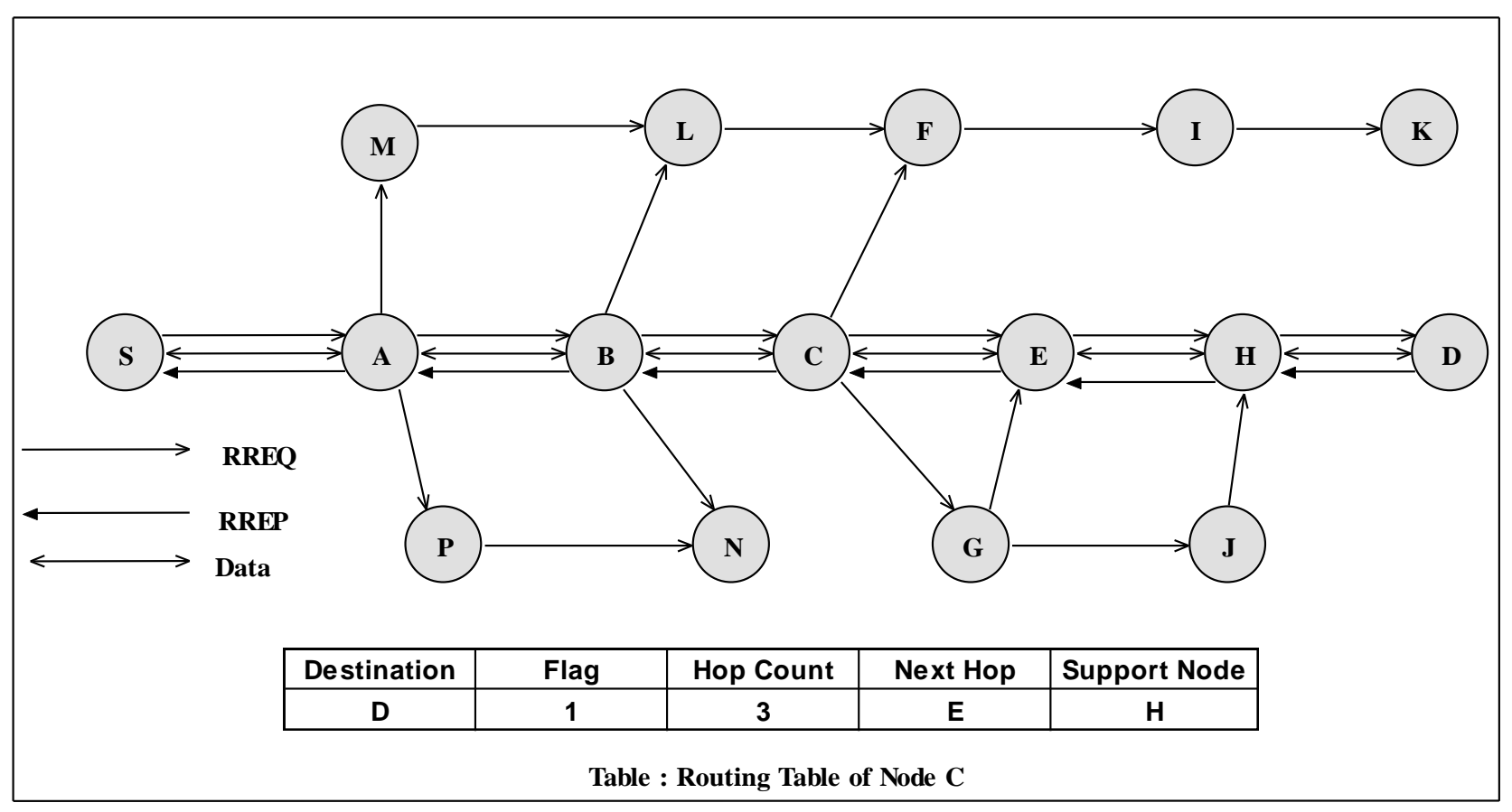

Fig.1. Route Construction

\section{B. Route Maintenance}

Due to high node mobility, if there is any link failure or an upstream node is not able to receive HELLO messages continuously for a fixed time interval, then it will assume that the link was broken. When a link failure was detected by a particular node on the established path, it will perform two actions.
1. Store the data and send a notification to previous nodes.

2. Recover route Failure.

For example in Fig.2, node $\mathrm{C}$ detects the link failure between node $\mathrm{C}$ and node $\mathrm{E}$. Then it will perform the above mentioned two actions.

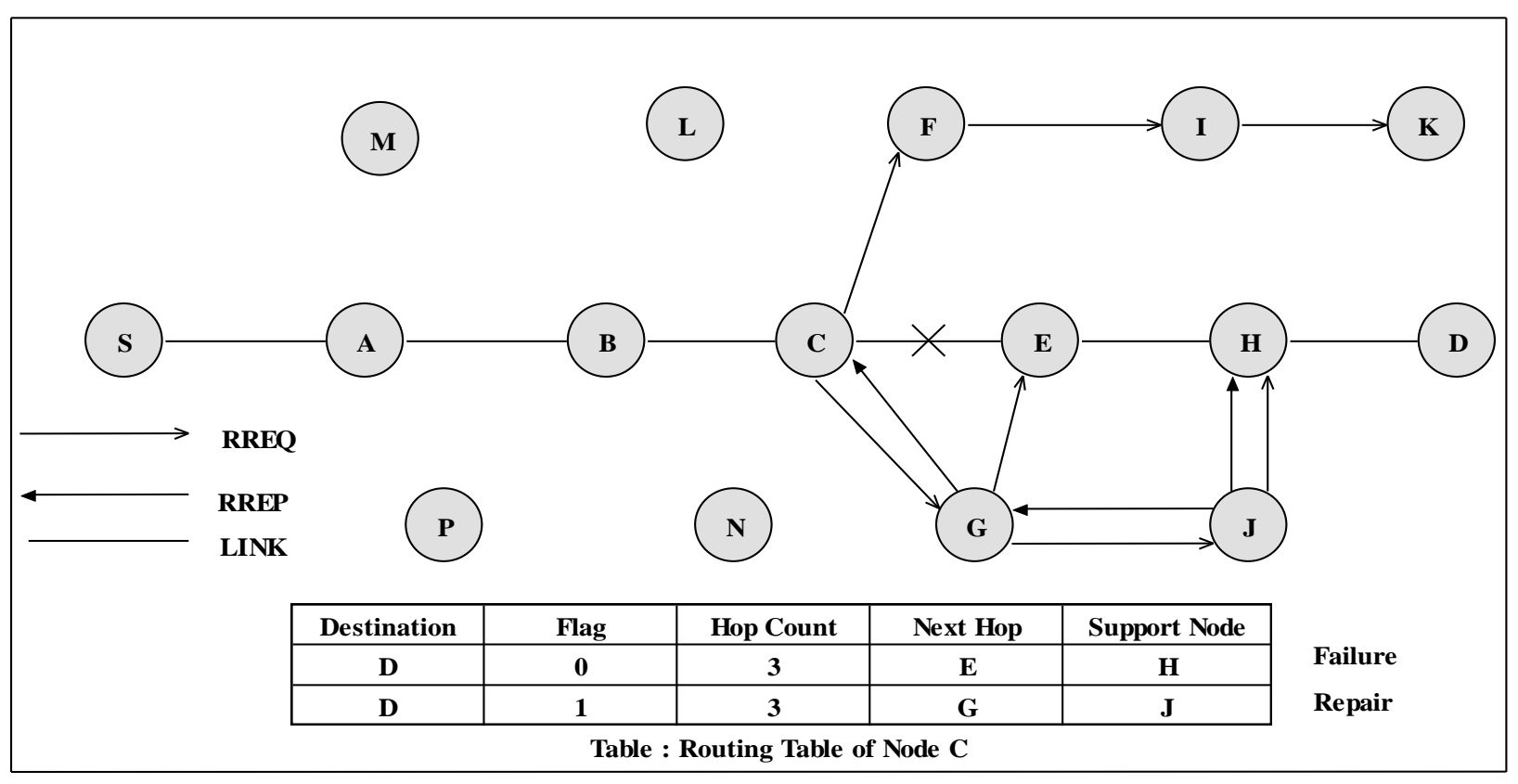

Fig.2. Route Recovery 
After the detection of a route failure at any intermediate node, it stores the complete data of eroded route in its buffer. The eroded data will be stored up to a fixed level of local buffer, fixed refers to $70 \%$ storage of node's buffer storage and it will transmit the buffered information after the route recovery. When the buffer was overloaded, an RREP of the same destination was forwarded to its one hop neighbor by indicating flag value as 1 . After the reception of this RREP by previous upstream node, it stops the data forwarding and starts to store the data in its local buffer. In this period, the node sets the state of flag as 2 , repair. The same process will be continued for other upstream nodes until the RREP packet reaches to source node. After receiving RREP at source node, a new route will be discovered towards the destination. In the Fig.2, node $\mathrm{C}$ stores the data in buffer up to its maximum level and then notifies to $\mathrm{B}$. The node at which the link failure was detected will broadcasts the Route Request Message (RRM) with the same destination address and also the support nodes address by keeping the transmission time interval (TTL) to 2. Upon receiving RRM, the intermediate nodes will perform the route construction process. The current source node receives the route reply message and forwards it to another onehop neighbor node to update the address of the support node of the previous upstream node. Intermediate nodes of an active route that have the routing information with a routing flag value set to the repairable state mentioned in Table 1 measure its own buffered data.

The routing flag of the routing table is set to the active state when the estimated buffer occupancy level goes below the already predefined buffer level. In Fig.2, node C broadcasts RRM towards destination D with support node $\mathrm{H}$ to repair the failed route between node $\mathrm{C}$ and node $E$. Node $G$ and $F$ receives the route request message and checks for a route to the support node and rebroadcasts the RRM because they don't find any route and also the PR metric is above the predefined threshold. In this way the RRM broadcasts and finally reaches to support node $\mathrm{H}$ through node $\mathrm{J}$ and then support node $\mathrm{H}$ replies to upstream node $\mathrm{C}$. Thus, a local route $\mathrm{C}-\mathrm{G}-\mathrm{J}-\mathrm{H}$ will establish and node $\mathrm{C}$ starts to forward the data to destination D. The algorithm 1 represents the route request handling during route discovery phase. The algorithm 2 represents the route maintenance phase in the case of route failure.

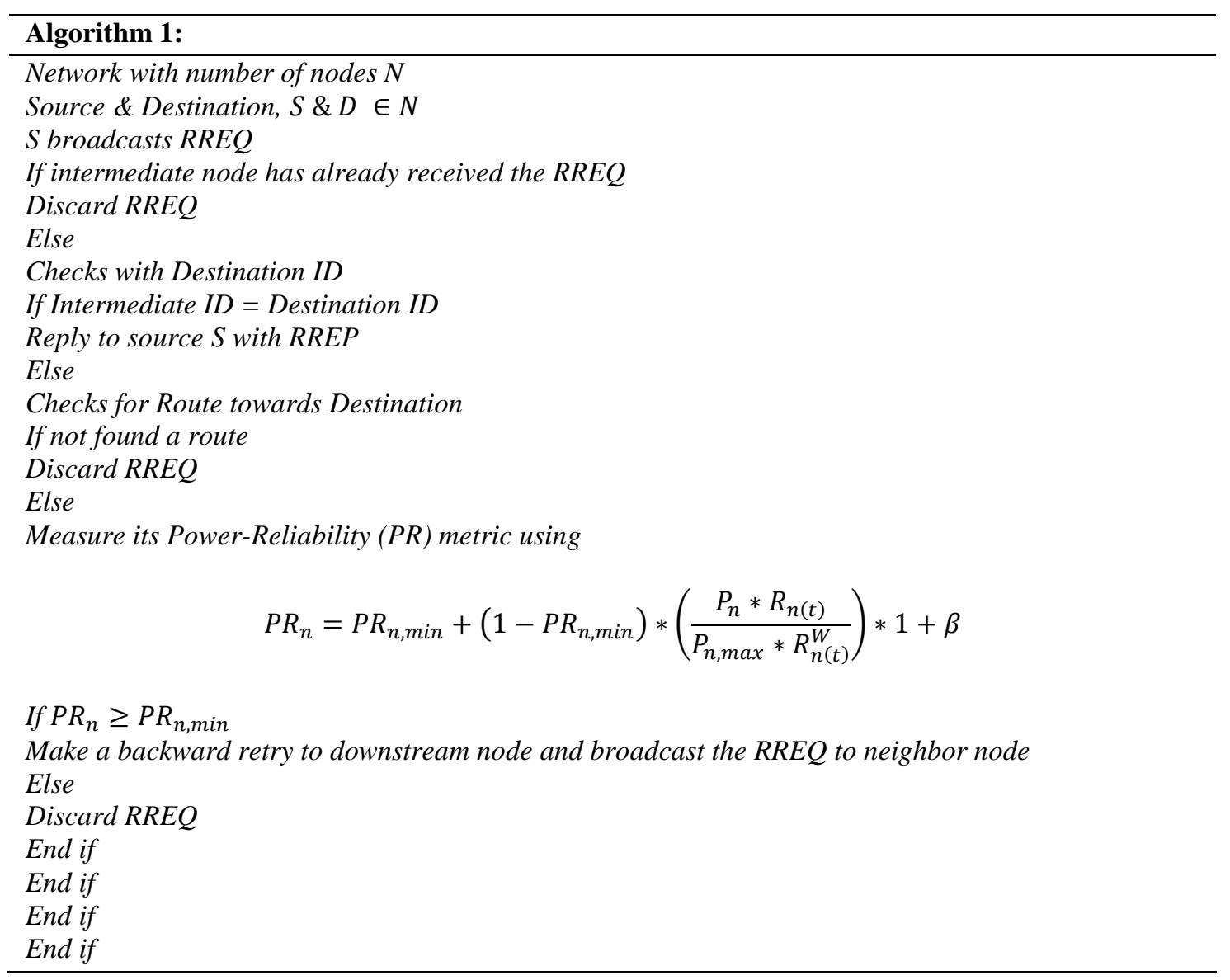




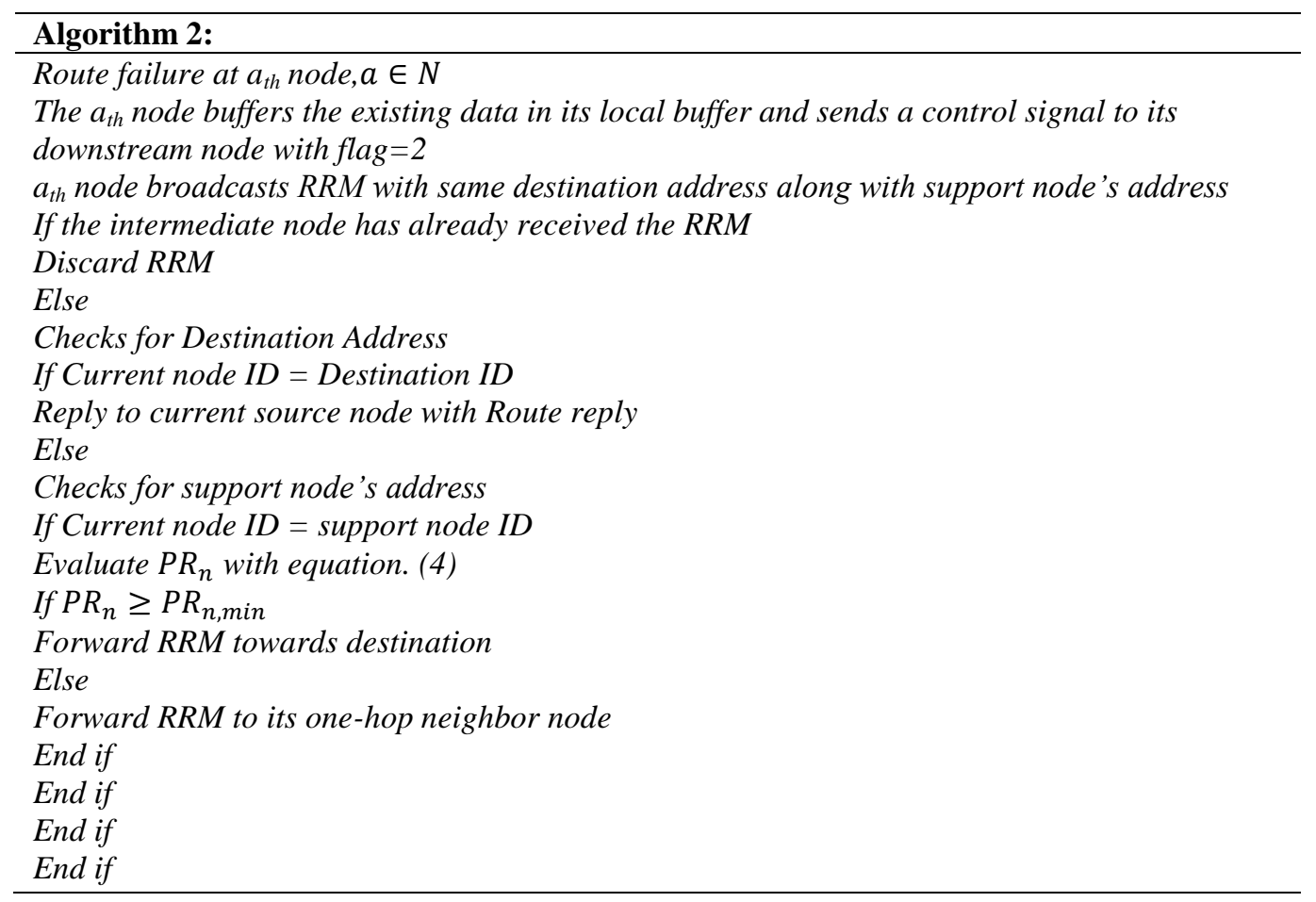

\section{Simulation RESULtS}

In this section, the performance analysis was represented through energy consumption, packet delivery ratio and end-to-end delay. The results of proposed approach were compared with various earlier approaches with varying mobility and packet size. The performance was measured for AODV [5], AODV-PE [17], EEPR [21] and the proposed PERR routing approaches by varying node motilities from $0 \mathrm{~m} / \mathrm{s}$ to $25 \mathrm{~m} / \mathrm{s}$. The simulation parameters are shown in Table.2.

Table 2. Simulation Parameters

\begin{tabular}{|c|c|}
\hline Configuration & Parameter Values \\
\hline Simulation Area & $1000 \mathrm{~m} \mathrm{X} 1000 \mathrm{~m}$ \\
\hline Number of Nodes & 50 \\
\hline Mobility Speed & $0,5,10,15,20,25 \mathrm{~m} / \mathrm{s}$ \\
\hline Source-Destination Pairs & 15 \\
\hline Packet Size & 512 bytes \\
\hline CBR Rates & 4 packets $/ \mathrm{sec}$ \\
\hline Mobility Model & RWP \\
\hline Pause Time (sec) & 60 \\
\hline
\end{tabular}

Average Energy Consumption: The total average amount of energy consumed for a successful delivery of packet is termed as average energy consumption. Though there are route failures, the proposed approach consumes less energy due to the route reconstruction criterion proposed.

Packet Delivery Ratio: The packet delivery ratio is the ratio of number of packets received at destination node to the number of packets transmitted from source node.

End-to-End Delay: it is defined as the time taken by a packet to reach to the destination.
Average energy consumption for varying node speed and for varying packet size was shown in Fig.3 and Fig.6 respectively where the proposed PERR having less average energy consumption compared with other approaches. Even for high number of route failures of high dynamic mobile environment, the PERR consumes less energy since it avoid the nodes which have too lower power level.



Fig.3. Average Energy Consumption for Varying Mobility speed in m/s

Due to node mobility, there is a frequent occurrence of link failures in MANETs. Packets will be dropped due to the frequent occurrence of link failures in all other protocols. The proposed PERR achieved better performance because of having on-demand local route recovery mechanism and data buffering capability, which significantly reduces the packet dropping rate. The Packet delivery ratio performance for varying node speed and for varying packet size was represented in Fig.4 and Fig.7 respectively. 




Fig.4. Packet Delivery Ratio for Varying Node Speed

The end-to-end delay variations of PERR for varying node speed and for varying packet size was shown in Fig.7 and in Fig.10 respectively. Since the PERR selects a route with small hop count and higher life time, the end-to-end delay will be less. From Fig.7, it is observed that, the end-to-end delay increases as the node speed increases. Though the delay having incremental characteristics, when PERR was compared with earlier approaches the delay of it is observed as less.



Fig.5. End-to-End Delay for Varying Node Speed

Fig.6, Fig.7 and fig.8 illustrate the performance of PERR through average energy consumption, packet delivery ratio and End-to-End delay respectively by varying packet size. Here, the varying packet size reflects to multimedia data packets, because, the multimedia information having large packet size. The proposed approach was also verified over multimedia data transmission and the obtained results declare that the proposed PERR outperforms other protocols even for multimedia data transmission. The simulation parameters for varying packet size in shown in Table.3.
Table 3. Simulation Parameters

\begin{tabular}{|c|c|}
\hline Configuration & Parameter Values \\
\hline Simulation Area & $1000 \mathrm{~m}$ X $1000 \mathrm{~m}$ \\
\hline Number of Nodes & 50 \\
\hline Mobility Speed & $25 \mathrm{~m} / \mathrm{s}$ \\
\hline Source-Destination Pairs & 15 \\
\hline Packet Size & $500-1000$ Bytes \\
\hline Mobility Model & RWP \\
\hline Pause Time (sec) & 60 \\
\hline
\end{tabular}

Fig.6 represents the average energy consumption by varying packet size. As the packet size increases, the amount of energy required also increases. Though the packet size increases the proposed approach utilizes less amount of average energy for forwarding the total packets, because the proposed approach evaluate a path with minimum energy consumption and also with minimum number of nodes. The proposed approach considered the help of support nodes thus the PERR having minimum energy consumption compared with earlier approaches.



Fig.6. Average Energy Consumption for Varying Packet Size

The details of packet delivery ratio for with varying packet size is represented in Fig.7.



Fig.7. Packet Delivery Ratio for Varying Packet Size 
From the Fig.7, the packet delivery ratio of PERR is observed to be high compared with earlier approaches, because, PERR considered the reliability during the route discovery and support nodes during route maintenance.

As the packet size increases, the amount of time taken for forwarding also increases. Fig. 8 represents the details of End-to-End delay for varying packet size for earlier and proposed approaches. Though there is an increased delay for increment in packet size, the PERR has less End-to-End delay compared with earlier approaches. Since the PERR forwards the information through support nodes, the delay is less compared with AODV, AODV-PE and EEPR.

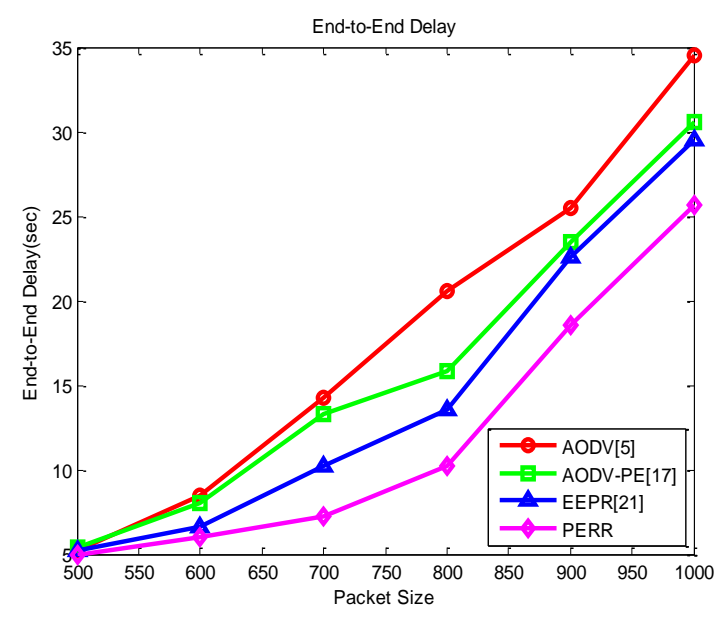

Fig.8. End-to-End Delay for Varying Packet Size

\section{CONCLUSIONS}

This paper proposed a power efficient routing and also an on-demand local route recovery mechanism for consistent data delivery in mobile Adhoc networks. PERR find most optimum route in terms of reliability, power efficiency and hop count. Reliability directs to reduce the packet dropping probability and increases packet delivery ratio. Power efficiency direct towards minimum energy consumption and less hop count directs to minimum delay. Simulation results showed the better performance of PERR through average energy consumption, packet delivery ratio and end-to-end delay over varying node speed and varying packet size. This approach showed the resource efficiency of network.

\section{REFERENCES}

[1] C. E. Perkins. Mobile Ad hoc Networking. AddisonWesley, Upper Saddle River, NJ, USA, 2001.

[2] Perkins C, Bhagwat P, "Highly dynamic destination sequenced distance-vector routing (DSDV) for mobile computers", Computer Communications Review, 1994, pp- 234-244.

[3] Pei G, Gerla M, Chen T-W, "Fisheye state routing: a routing scheme for ad hoc wireless networks", Proceedings of IEEE International Conference on Communications (ICC), 2000, pp.70-74.

[4] Johnson D, Maltz D, "Dynamic source routing in ad hoc wireless networks", book Mobile Computing, edited by Tomasz Imielinski and Hank Korth, Kluwer Academic Publishers, 1996, pp.153-181.

[5] Perkins C, Royer E, 'Ad-hoc on-demand distance vector routing', Proceedings of 2nd IEEE Workshop on Mobile Computing Systems and Applications, 1999, pp.90-100.

[6] S.-J. Lee and M. Gerla, "AODV-BR: Backup routing in ad hoc networks," in Proceedings of the IEEE Wireless Communications and Networking Conference (WCNC '00), IEEE, Chicago, Ill, USA, September 2000 pp. 1311-1316.

[7] W. K. Lai, S.-Y. Hsiao, and Y.-C. Lin, "Adaptive backup routing for ad-hoc networks," Computer Communications, vol. 30, no. 2, 2007, pp. 453-464.

[8] J. Jeon, K. Lee, and C. Kim, "Fast route recovery scheme for mobile ad hoc networks," in Proceedings of the International Conference on Information Networking (ICOIN'11), January 2011, pp. 419-423.

[9] M. M. R. Bosunia, M. A. Razzaque, and M. M. Islam, “A robust and reliable routing protocol for energyconstrained mobile ad hoc networks," International Journal of Computing Communication and Networking Research, vol. 2, no. 2, 2013, pp. 26-39.

[10] M. Aparna, M. Reza, P. Sahu, and S. Das, "An efficient approach towards robust routing in MANET," in Proceedings of the International Conference on Communication Systems and Network Technologies (CSNT '12), IEEE, Rajkot, India, May2012, pp. 388-391.

[11] T. T. Son, H. L. Minh, G. Sexton, and N. Aslam, "A novel encounter-based metric for mobile ad-hoc networks routing," Ad Hoc Networks, vol. 14,2014, pp. 2-14.

[12] T. C. J. Boleng and W. Navidi, "Metrics to enable adaptive protocols for mobile ad hoc networks," in Proceedings of the International Conference on Wireless Networks (ICWN '02), Las Vegas, Nev, USA, June 2002, pp.293-298.

[13] C. Yawut, B. Paillassa, and R. Dhaou, "Mobility metrics evaluation for self-adaptive protocols," Journal of Networks, vol. 3, no.1, 2008, pp. 53-64.

[14] F. Ingelrest, N. Mitton, and D. Simplot-Ryl, “A turnover based adaptive hello protocol for mobile ad hoc and sensor networks," in Proceedings of the 15th International Symposium on Modeling Analysis, and Simulation of Computer and Telecommunication Systems (MASCOTS '07), October 2007, pp. 9-14.

[15] K. Woo, C. Yu, D. Lee, H. Y. Youn, and B. Lee, "Nonblocking, localized routing algorithm for balanced energy consumption in mobile ad hoc networks," in Proceedings of the 9th International Symposium in Modeling, Analysis and Simulation of Computer and Telecommunication Systems (MASCOTS '01), p.117, IEEE Computer Society, Washington, DC, USA, 2001.

[16] Z. Zhaoxiao, P. Tingrui, and Z.Wenli, "Modified energyaware AODV routing for ad hoc networks," in Proceedings of the WRI Global Congress on Intelligent Systems (GCIS '09), vol. 3,May 2009 pp. 338-342.

[17] C. Ni, T. Lee, G. Kim, and C. Kim, "An energy aware routing protocol using multiple replies for ad hoc networks," in Proceedings of the 2nd ISECS International Colloquium on Computing, Communication, Control, and Management (CCCM '09), August 2009, pp.225-228.

[18] G.M. Rao, M. N. Baig, M. F. Baba, and K. K. Kumar, "Energy efficient reliable routing protocol for mobile ad hoc networks," in Proceedings of the 3rd International Conference on Electronics Computer Technology (ICECT '11), Kanyakumari, India, April 2011, pp. 296299. 
[19] X. Wang, L. Li, and C. Ran, "An energy-aware probability routing in MANETs," in Proceedings of the IEEE Workshop on IP Operations and Management, October 2004, pp. 146-151.

[20] P. Nand and S. C. Sharma, "Probability based improved broadcasting for AODV routing protocol," in Proceedings ofthe International Conference on Computational Intelligence and Communication Systems (CICN' 11), October 2011, pp. 621-625.

[21] S.-H. Park, S. Cho, and J.-R. Lee, "Energy-efficient probabilistic routing algorithm for internet of things," journal of Applied Mathematics, vol. 2014, Article ID 213106, 7 pages.

[22] Jinhua Zhu and Xing Wang, "Model and Protocol for Energy Efficient Routing over Mobile Adhoc Networks", IEEE Transaction on Mobile Computing, Volume.10, No.11, November 2011.

\section{Authors' Profiles}

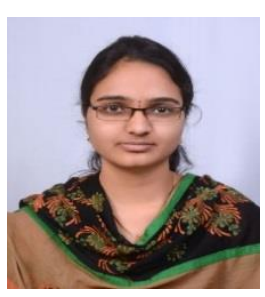

Vallala Sowmya Devi is currently a PHD candidate at JNT University, Hyderabad, Telangana, India and she is also working as an Assistant Professor at the Department of Computer Science and Engineering in GITAM University, Hyderabad, and Telangana, India. She has eight years of teaching experience.
She has done B.Tech and M.tech in Computer Science and Engineering from JNTUH affiliated engineering college. Her current research interests include wireless networks, mobilecommunications, network security, information security and MANETs.



Dr. Nagaratna P. Hegde is a professor of Computer Science and Engineering Department at Vasavi College of Engineering, Hyderabad, and Telangana, India. She received her doctorate in Computer Science \& Engineering from JNTU in 2009. She has published more than 30 well researched papers. Her research interests include Cellular automata, Spatial Simulation modeling, GIS-Software, Neural Network, Artificial Intelligence.

How to cite this paper: V.Sowmya Devi, Nagaratna P Hegde,"Reliable and Power Efficient Routing Protocol for MANETs", International Journal of Computer Network and Information Security(IJCNIS), Vol.8, No.10, pp.61-69, 2016.DOI: $10.5815 /$ ijcnis.2016.10.08 